\title{
Development of GABARAP family protein- sensitive LIR-based probes for neuronal autophagy
}

\author{
Pureum Jeon ${ }^{1 \dagger}$, Ju-Hui Park ${ }^{2 \dagger}$, Yong-Woo Jun², You-Kyung Lee ${ }^{1}$, Deok-Jin Jang ${ }^{2^{*}}$ and Jin-A Lee ${ }^{1 *}$
}

\begin{abstract}
Autophagy allows for lysosomal cellular degradation of cytosolic components. In particular, neuronal autophagy is essential for cellular homeostasis and neuronal survival and is tightly regulated by several autophagy-related (ATG) proteins in post-mitotic neurons. Among these ATG proteins, the LC3/GABARAP proteins are known to regulate autophagosome biogenesis/maturation and cargo recognition. However, little is known about the role of GABARAP family proteins in neuronal autophagy despite their abundant expression in post-mitotic neurons. We have previously developed HyD (Hydrophobic Domain)-LIR (LC3-interacting region)-based autophagosome markers. In this study, to monitor GABARAP family proteins in autophagosomes of post-mitotic neurons, we improved the sensitivity of the probes for specifically detecting endogenous GABARAP family proteins by adding one more LIR motif to the LIR probes. We have tested the efficiency of two different LIRs, from ULK2 and Stbd1, in regard to their cellular localization to autophagosomes. HyD-2xLIR(ULK2)-GFP and HyD-2xLIR(Stbd1)-GFP demonstrated specific localization to GABARAP-positive autophagosomes relative to LC3B-positive autophagosomes in MEF/HeLa cells in an autophagy-dependent manner. Indeed, HyD-2xLIR(Stbd1)-GFP could efficiently detect GABARAP-positive autophagosomes in cultured cortical neurons. Our improved GABARAP-sensitive probes will contribute toward understanding the specific role of GABARAP family proteins in regard to neuronal autophagy.
\end{abstract}

Keywords: LC3, GABARAP, LIR motif, Autophagy

\section{Main text}

Autophagy is a highly regulated cellular pathway involved in lysosomal degradation of unnecessary and/or dysfunctional cellular components wrapped by a double-membrane-bound autophagosome within the cells [1]. In a highly polarized neuron, neuronal autophagy is essential for cellular homeostasis and cell survival under physiological and pathological conditions due to its post-mitotic nature and dynamic signaling at the synapse [2]. Among the many ATG (AuTophagy-related Gene) genes, the ATG8 protein is involved in autophagosome formation, cargo recognition and recruitment to

\footnotetext{
* Correspondence: jangdj@knu.ac.kr; leeja@hnu.kr

${ }^{\dagger}$ Pureum Jeon and Ju-Hui Park contributed equally to this work.

${ }^{2}$ Department of Ecological Science, College of Ecology and Environment, Kyungpook National University, 386, Gajang-dong, Sangju-si, Kyungbuk 37224, South Korea

'Department of Biological Sciences and Biotechnology, College of Life Sciences and Nanotechnology, Hannam University, 461-6 Jeonmin-dong, Yuseong-gu, Daejeon 34054, Republic of Korea
}

autophagosomes. In human cells, at least seven ATG8 homologues (mammalian ATG8, mATG8) are expressed. These are generally divided into the two subfamilies of GABARAP proteins, including GABARAP/-L1/-L2 as well as $\mathrm{LC} 3$, which includes $\mathrm{LC} 3 \mathrm{~A}(\mathrm{a}, \mathrm{b}) / \mathrm{B} / \mathrm{C}$. Interestingly, the GABARAP family proteins, relative to the LC3 proteins, are localized to the initial segment of axons as well as being present in synaptic-vesicle-enriched fractions [3]. However, the differential roles of each LC3/ GABARAP family protein in neuronal autophagy or selective autophagy are largely unknown. In addition, the ability to detect LC3/GABARAP proteins in polarized neurons is limited, as overexpression of LC3 family proteins can cause abnormal branching or induced protein aggregation in an autophagy independent manner [4].

To overcome these issues, we have recently developed new autophagosome sensors to detect endogenous LC3/ GABARAP proteins in autophagosomes using LIR (LC3-interacting region) motifs and a hydrophobic motif

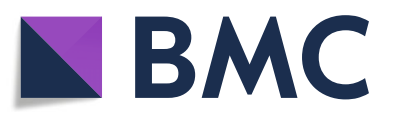

C The Author(s). 2019 Open Access This article is distributed under the terms of the Creative Commons Attribution 4.0 International License (http://creativecommons.org/licenses/by/4.0/), which permits unrestricted use, distribution, and reproduction in any medium, provided you give appropriate credit to the original author(s) and the source, provide a link to the Creative Commons license, and indicate if changes were made. The Creative Commons Public Domain Dedication waiver (http://creativecommons.org/publicdomain/zero/1.0/) applies to the data made available in this article, unless otherwise stated. 
(HyD) [4]. In our previous study, among the LIRs identified from different LIR-containing proteins, some of these domains demonstrated selective GABARAP family binding, but these were weakly associate with GABARAP-positive autophagosomes. Therefore, to improve the efficiency of these HyD-LIR-GFP sensors for monitoring GABARAP proteins, we generated HyD2xLIR-GFP sensors by duplicating the LIR motifs from selective GABARAP family binding proteins, including LIR motifs from the ULK2 and Stbd1 proteins, into HyD-GFP (Fig. 1a).
To compare the efficiency of HyD-LIR-GFP and HyD-2xLIR-GFP in regard to cellular localization to LC3/GABARAP-positive autophagosomes, we transfected HyD-1xLIR-GFP or HyD-2xLIR-GFP together with each mRFP-LC3/GABARAP family protein into MEF cells incubated with rapamycin (100 nM, 4h), a general autophagy inducer, under inhibition of lysosomal degradation $\left(10 \mathrm{mM} \mathrm{NH}_{4} \mathrm{Cl}, 4 \mathrm{~h}\right)$. As shown in Fig. $1 b$ and $c$, the HyD-2xLIR-GFP sensors could more efficiently detect GABARAP-positive autophagosomes when compared to HyD-LIR-GFP, indicating that the duplication

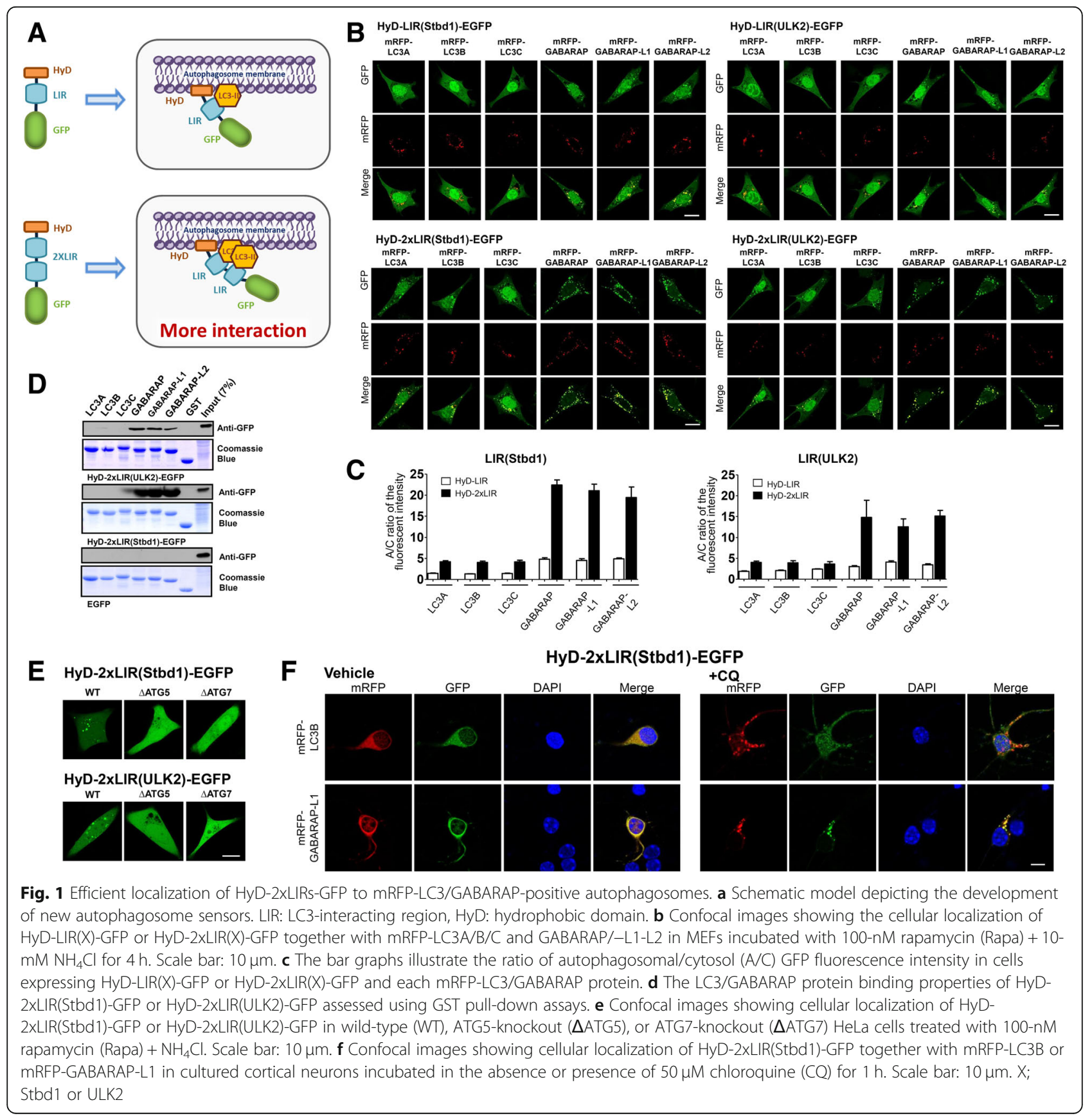


of the LIR motif from ULK2 or Stbd1 could generally enhance its cellular localization to GABARAP-positive autophagosomes. These results indicated that either HyD-2xLIR(ULK2)-GFP or HyD-2xLIR(Stbd1)-GFP could be used as sensors for detecting GABARAP-positive autophagosomes.

To further validate the binding preference of each HyD-2xLIR-GFP marker, we performed GST pulldown assays with each GST-LC3/GABARAP protein. As shown in Fig. 1d, HyD-2xLIR(ULK2)-GFP and HyD-2xLIR(Stbd1)-GFP each bound selectively to GST-GABARAP/-L1-L2, which was consistent with the efficacy of its cellular targeting to GABARAP -positive autophagosomes.

We also overexpressed HyD-2xLIR(ULK2)-GFP or HyD-2xLIR(Stbd1)-GFP in ATG5 and ATG7-knockout HeLa cells. Both HyD-2xLIR(ULK2)-GFP and HyD2xLIR(Stbd1)-GFP could detect vesicle structures in wild-type HeLa cells but not in ATG5 or ATG7-knockout HeLa cells (Fig. 1e), suggesting that our improved probes specifically detected endogenous GABARAP-positive autophagosomes in living cells.

Finally, we tested whether our improved LIR-based sensors could detect neuronal autophagosomes in neurons expressing mRFP-LC3B or mRFP-GABARAP-L1. To accomplish this, HyD-2xLIR(Stbd1)-GFP together with either mRFP-LC3B or mRFP-GABARAP were transfected into cortical neurons. Indeed, HyD-2xLIR(Stbd1)-GFP localized to mRFP-GABARAP-L1-positive autophagosomes but not to mRFP-LC3B-positive autophagosomes in the presence of a lysosomal inhibitor (Fig. 1f). These results suggested that HyD-2xLIR(Stbd1)-GFP functions as a useful marker for monitoring GABARAP-positive autophagosomes in neurons.

To develop a fluorescence-based LC3/GABARAP-specific sensor, our group and Dr. Ivan's group have generated LIR-based autophagosome sensors [4, 5]. Our group used $\mathrm{HyD}$ to enhance membrane association while Ivan's group used a membrane recruitment (FYVE) or oligomerization (PB1) domain. Specifically, in neurons expressing aggregate-prone proteins, overexpressed LC3 or GABARAP might associate with aggregate-prone proteins in an autophagy-independent manner [6, 7]. Therefore, the specific HyD-LIR sensors detailed in this report will be highly useful for the study of autophagy based on their ability to monitor endogenous LC3- or GABARAP-positive autophagosomes.

\section{Abbreviations}

ATG: AuTophagy-related Gene; GABARAP: Gamma-aminobutyric acid receptor-associated protein; HyD: Hydrophobic domain; LIR: LC3-interacting region

\section{Funding}

This work was supported by the National Research Foundation (NRF2017R1D1A3B03030972), the National Scientist Honor Program, the Korea Health Technology R\&D Project (HI18C0158), and the Bio \& Medical

Technology Development Program of the National Research Foundation (NRF) funded by the Ministry of Science \& ICT (2017M3A9G7073521) to J.-A L. and by the Basic Science Research Program through the NRF (NRF-

2018R1D1A1B07048822) to D.-J.J.

\section{Availability of data and materials}

All data generated or analyzed during this study are included in this published article.

\section{Authors' contributions}

$J A L$ and DJJ designed the study, analyzed the data, and wrote the paper. PJ, $J H P, Y K L$ and YWJ performed the experiments. All authors read and approved the final manuscript.

Ethics approval and consent to participate

Not applicable.

Consent for publication

Not applicable.

\section{Competing interests}

The authors declare that they have no competing interests.

\section{Publisher's Note}

Springer Nature remains neutral with regard to jurisdictional claims in published maps and institutional affiliations.

Received: 22 March 2019 Accepted: 1 April 2019

Published online: 08 April 2019

References

1. Levine B, Kroemer G. Biological functions of autophagy genes: a disease perspective. Cell. 2019;176:11-42.

2. Nikoletopoulou $\mathrm{V}$, Tavernarakis N. Regulation and roles of autophagy at synapses. Trends Cell Biol. 2018;28:646-61.

3. Koike M, Tanida I, Nanao T, Tada N, Iwata J, Ueno T, Kominami E, Uchiyama $Y$. Enrichment of GABARAP relative to $L C 3$ in the axonal initial segments of neurons. PLoS One. 2013;8:e63568.

4. Lee YK, Jun YW, Choi HE, Huh YH, Kaang BK, Jang DJ, Lee JA. Development of LC3/GABARAP sensors containing a LIR and a hydrophobic domain to monitor autophagy. EMBO J. 2017;36:1100-16.

5. Stolz A, Putyrski M, Kutle I, Huber J, Wang C: Fluorescence-based ATG8 sensors monitor localization and function of LC3/GABARAP proteins. 2017, 36:549-564.

6. Klionsky DJ, Abdelmohsen K, Abe A, Abedin MJ, Abeliovich H, Acevedo Arozena A, Adachi H, Adams CM, Adams PD, Adeli K, et al. Guidelines for the use and interpretation of assays for monitoring autophagy (3rd edition). Autophagy. 2016;12:1-222.

7. Kuma A, Matsui M, Mizushima N. LC3, an autophagosome marker, can be incorporated into protein aggregates independent of autophagy: caution in the interpretation of LC3 localization. Autophagy. 2007;3:323-8.

Ready to submit your research? Choose BMC and benefit from:

- fast, convenient online submission

- thorough peer review by experienced researchers in your field

- rapid publication on acceptance

- support for research data, including large and complex data types

- gold Open Access which fosters wider collaboration and increased citations

- maximum visibility for your research: over $100 \mathrm{M}$ website views per year

At BMC, research is always in progress.

Learn more biomedcentral.com/submission 